\title{
REVIEWERS
}

\section{Acknowledgment of Reviewers for Volume 8}

Published online: 3 November 2015

(C) Springer International Publishing 2015

We would like to acknowledge the following peer reviewers who have dedicated their time and have contributed to the quality of this journal throughout the production of Volume 8:

Judie Alpert

Mary-Jo Bautista

Greg Bohall

Elspeth Bradley

Ernestine Briggs-King

Courtenay Cavanaugh

Elizabeth Colantuoni

Delphine Collin-Vezina

Shir Daphna-Tekoah

Anne DePrince

Anders Dovran

Grete Dyb

Marc Felizzi

David Finklehor

James Garbarino

Kelly Graves

David Griffin

Jessica Griffin

Michelle Harriman

Martine Hebert

H. Alan Heisterkamp
Philip Ikomi

Maureen Kenny

Mona Khoury-Kassabri

Ibrahim Kira

David Lawson

Rebecca T. Leeb

Gregory Lewis

Diane Purvin

Maryse Richards

Rebecca Sanderson

Cris Scaglione

Jenelle Shanley

Roberta Sprague

Enrico Suardi

Pratyusha Tummala-Narra

Monica Ulibarri

Emilio Ulloa

Bessel van der Kok

Debra Warner

Ineke Way

Michael Weuste 